\title{
Cuerpo-territorio y aborto: propuesta teórica para cuestionar la gobernanza reproductiva violenta ${ }^{1}$
}

\author{
Body-Territory and Abortion: A Theoretical Approach \\ to Question Violent Reproductive Governance
}

Fecha de recepción: 24/08/2021

Fecha de aceptación: 24/11/2021

Fecha de publicación: 07/03/2022

https://doi.org/10.48102/if.2022.v2.n1.194

\author{
Madeleine Belfrage* \\ m.belfrage@uq.edu.au \\ ORCID: https://orcid.org/0000-0001-5331-3690 \\ Maestra en Práctica del Desarrollo \\ University of Queensland \\ Australia
}

\begin{abstract}
* Es candidata al doctorado en Ciencias Sociales y maestra en Práctica del Desarrollo por la Universidad de Queensland, Australia. Es integrante del consejo de la Red Internacional por la Reducción del Estigma y Discriminación del Aborto (Inroads, por sus siglas en inglés) y de la coordinación de la Asociación por los Estudios Ibéricos y Latinoamericanos de Australia (AILASA, por sus siglas en inglés). Además, es coautora de "Story Circles and abortion stigma in Mexico: a mixed-methods evaluation of a new intervention for reducing individual level abortion stigma" (Belfrage et al., 2019) y "Failing forward: A case study in neoliberalism and abandonment in Calama" (Babidge y Belfrage, 2017). Sus áreas de interés son aborto autónomo, estigma, feminismos descoloniales latinoamericanos, gobernabilidad reproductiva y neoliberalismo.
\end{abstract}

1 Este artículo contiene extractos de su trabajo de tesis, la cual se encuentra en desarrollo. 


\section{Resumen}

En este ensayo, exploro la forma en la que, en México, el acompañamiento feminista para mujeres que necesitan realizarse un aborto se enmarca en agendas más amplias relacionadas con la autonomía corporal y la violencia de género. A través de narraciones derivadas de entrevistas etnográficas con acompañantes, demuestro cómo se desarrollan las luchas de poder a través de territorios somáticos, mientras las feministas luchan contra los regímenes violentos de gobernanza reproductiva que controlan los cuerpos de las mujeres. Así, utilizo el marco analítico descolonial feminista latinoamericano del cuerpo-territorio, con el fin de conceptualizar las luchas actuales por la autonomía corporal, más allá del conocido y constantemente enunciado "derecho a decidir", en un contexto en el que de forma paradójica los cuerpos de las mujeres están sobrerregulados y de manera simultánea son violados y abandonados por el Estado y los actores estatales paralelos.

\section{Palabras clave}

Acompañamiento, aborto, cuerpo-territorio, gobernanza, necropolítica.

\section{Abstract}

In this essay, I explore how, in Mexico, feminist accompaniment for women in need of abortion is framed by broader agendas related to bodily autonomy and gender-based violence. Using narratives drawn from ethnographic interviews with accompaniers, I demonstrate how power struggles play out across somatic territories as feminists struggle against violent regimes of reproductive governance that control women's bodies. I use the Latin American feminist decolonial analytical framework of the body-territory to conceptualize current struggles for bodily autonomy, beyond the well-known and constantly enunciated "right to decide," in a context in which women's bodies are paradoxically over-regulated and simultaneously violated and abandoned by the State and parallel State actors.

\section{Keywords}

Accompaniment, abortion, body-territory, governance, necropolitics. 


\section{Introducción}

Ciudad de México, 8 de marzo de 2020. Desde la desaparición de los 43 estudiantes de Ayotzinapa en 2014, las calles de la Ciudad de México nunca habían estado tan llenas de cuerpos que gritan y marchan. En esa ocasión, la mayoría fueron mujeres: jóvenes, ancianas, indígenas, morenas, cis, trans, lesbianas, bisexuales, gordas, delgadas, con discapacidades y sin ellas.

Mientras garabateaba notas en mi cuaderno, alerta para no estorbar cuando pasaran las anarquistas con martillos, las pancartas llamaron mi atención. Entre la multitud, había carriolas, granadas de humo y brillantina; además, podían leerse frases como "Aborto Legal Ya", "Estado Violador"2 y "Narcoestado Feminicida". A partir de ese momento, me di cuenta de que era imposible entender las demandas feministas para la autonomía corporal en torno al aborto sin comprender el contexto de violencia nauseabunda contra las mujeres, el cual no se detiene.

Históricamente, en México, las demandas feministas por la autonomía corporal se han basado en el marco discursivo y normativo del "derecho a decidir" (Lamas, 2015), con el objetivo de que el Estado lo garantice. Así, muchas feministas han trabajado para despenalizar el aborto a nivel estatal y federal, mejorar las políticas de salud para brindar servicios de calidad para la interrupción del embarazo, reducir la burocracia legal y presionar a las autoridades y al personal de salud para cumplir con sus obligaciones.

Estas acciones se basan en un concepto del Estado como garante de derechos y proveedor de bienestar social. Sin embargo, los mensajes en las pancartas en las marchas feministas más recientes que refieren a un "Estado-violador" y a un "Narcoestado feminicida" apuntan a una visión diferente: la del Estado como un poder soberano que, en el sentido foucaultiano (Foucault, 1978), se aleja de proteger a la ciudadanía para gobernar a través del poder de la violencia y la muerte, ejerciendo la "necropolítica" (Mbembe, 2003). ${ }^{3}$

2 "Estado-violador" apunta a acusaciones hacia el Estado por su papel en la epidemia de violencia contra las mujeres; el caso más emblemático en el momento de esta investigación era la presunta violación de una adolescente por parte de policías en la Ciudad de México.

3 "Narcoestado" es un neologismo que se refiere a un Estado implicado y enriquecido por el narcotráfico. 
¿Cómo entender las demandas y acciones feministas sobre el aborto en un contexto violento, en donde el Estado ha sido ineficaz para proteger a las mujeres y además está implicado en la violencia contra sus cuerpos?

En este ensayo, exploro cómo puede conceptualizarse el acompañamiento feminista de las mujeres para realizar abortos, dentro y fuera de los sistemas de salud y las leyes estatales, desde una perspectiva feminista latinoamericana descolonial del cuerpo-territorio (Cabnal, 2010; Cruz Hernández, 2016; Paredes, 2008), que va más allá del concepto estatalista y hegemónico del aborto como "derecho a decidir". Presento una propuesta analítica y argumentativa que se sustenta en dos entrevistas etnográficas que realicé a acompañantes que trabajan en diferentes entornos: la primera, en comunidades del estado de Michoacán afectadas por la narcoviolencia; la segunda desarrolla sus actividades en hospitales públicos del Estado de México.

Las entrevistas se realizaron por medio de videollamadas y forman parte de una investigación más amplia, que constituye mi tesis doctoral y que tuvo una duración de dieciocho meses (de 2019 a 2020). Dicho trabajo se basa en la metodología etnográfica activista feminista (Craven y Davis, 2013): incluyó sesenta entrevistas a profundidad, así como observación participante con acompañantes en encuentros, reuniones, marchas y otros eventos realizados en línea debido a la contingencia de la pandemia de COVID-19. Asimismo, es fundamental mi experiencia de varios años de trabajo en organizaciones no gubernamentales $(\mathrm{ONG})$ feministas que capacitan y apoyan a acompañantes en México.

Soy consciente de mi posición como mujer blanca, cisgénero y heterosexual, nacida en Australia. En este sentido, intento descolonizar mi investigación al centrar mi análisis en las estructuras y sistemas de poder (Curiel, 2015) utilizando herramientas teóricas creadas por autoras y activistas feministas latinoamericanas. Lo anterior, con el debido cuidado y respeto para no apropiarme del conocimiento en un acto de colonización discursiva (Mohanty, 2003).

En este sentido, mi análisis se ubica en el marco de la gobernanza reproductiva, que incluye los mecanismos y las estructuras del Estado a través de los cuales se gobiernan los cuerpos reproductivos (Morgan y Roberts, 2012). Sostengo que, en el contexto mexicano, la gobernanza reproductiva es altamente violenta y que, de forma paradójica, los cuerpos de las mujeres son sobrerregulados y al mismo tiempo violentados, asesinados y abandonados por el Estado. 
El concepto "cuerpo-territorio" permite profundizar en el análisis de los ensamblajes del poder y la resistencia que se manifiestan en los cuerpos de las mujeres en las luchas actuales por la autonomía reproductiva en México. Al dejar de evocar ideas de individualismo liberal, implícitas en el concepto de "decisión", es posible conceptualizar la autonomía como la defensa de un territorio somático, en un contexto en el que los cuerpos son colonizados, controlados y eliminados como moneda de poder (Segato, 2008; Valencia, 2010).

Las narraciones de las acompañantes que presento en este ensayo demuestran cómo se utilizan los cuerpos de las mujeres para producir y mantener un territorio soberano mediante la violencia física y legislativa en torno al aborto. Asimismo, describo las formas en las que las activistas feministas desafían estas condiciones, a partir de la defensa encarnada del territorio, con la práctica autónoma del aborto y propiciando que el Estado rinda cuentas a través del acompañamiento en los hospitales públicos.

Este ensayo se conforma de tres partes: en primer lugar, coloco la discusión sobre el concepto cuerpo-territorio y cómo se relaciona con la gobernanza reproductiva, violenta e implícita en el control de los cuerpos de las mujeres. Además, describo el contexto legal del aborto en México como un ejercicio territorial en el que el Estado ejerce poder a través de una legislación violenta.

En un segundo momento, presento el caso de una acompañante feminista que decidió identificarse como Asunción y que es originaria del estado de Michoacán. A través de su narración, abordo la relación entre los cuerpos y el territorio, en un contexto en el que los abortos ocurren como producto de la narcoviolencia. Sostengo que las acompañantes buscan garantizar la autonomía reproductiva de las mujeres en entornos donde sus cuerpos son gobernados como una extensión del territorio, lo que se vincula con las luchas violentas entre el Estado y otros actores hostiles que se involucran en lo que Sayak Valencia (2010) denomina "necroempoderamiento": el consumo de los cuerpos como una práctica distópica mercantilizada, consecuencia de las precarias condiciones del denominado "Tercer Mundo", ${ }^{4}$ bajo el neoliberalismo.

4 Valencia (2010) utiliza el término "Tercer Mundo" de manera crítica para significar las condiciones económico-sociales de México, que son geopolíticamente influenciadas, y las "propias y distintas estrategias de empoderamiento" que resultan de las mismas (p. 205). 
En la tercera parte, retomo la experiencia de Esperanza, acompañante que apoya a las mujeres para abortar con servicios de salud públicos y dentro del marco legal. Así, exploro el papel del Estado al permitir o negar el acceso a la atención médica para el aborto como una especie de estado de excepción (Agamben, 1998). Sostengo que los cuerpos de las mujeres se gobiernan territorialmente a través de jurisdicciones que protegen arbitrariamente los derechos de algunas y los niegan a otras. Incluso en circunstancias en las que los abortos se consideran "legales", se niega de manera rutinaria la atención médica que salvaría la vida de las mujeres, lo que propicia una especie de necropolítica reproductiva.

Por lo tanto, afirmo que las dos acompañantes entrevistadas realizan su trabajo en contextos donde la noción de "decisión" puede parecer una anomalía y la de "derechos", un sueño utópico. De tal forma que teorizar el aborto como la defensa de un territorio corporal trastoca y profundiza los discursos hegemónicos para reflejar la complejidad de las demandas y acciones feministas de autonomía corporal en espacios de intensa y profunda violencia de género. El poder de las acciones de las acompañantes radica en la transformación de los cuerpos de las mujeres de víctimas a custodias corporales territoriales.

\section{Cuerpo-territorio y la gobernanza reproductiva}

Históricamente, las mujeres indígenas y feministas latinoamericanas acuñaron y articularon el concepto cuerpo-territorio para posicionarse en contra del extractivismo, la violencia estatal y el desplazamiento de sus comunidades (Cabnal, 2010; Cruz Hernández, 2016; Paredes, 2008). De esta forma, se apunta a la dinámica de los estados poscoloniales, cuya historia se basa en la conquista de los cuerpos y la geografía, en los que los procesos actuales de extracción y acumulación capitalista y globalizada se apropian de y exponen a los cuerpos a la violencia.

En este sentido, cuerpo-territorio es lo que Vargas (2017) asume como una teoría/experiencia/imaginario viajera que ha migrado al movimiento del aborto, en particular entre los colectivos feministas que practican el acompañamiento desde una perspectiva basada en la teoría descolonial. Como argumenta Yuderkys Espinosa (Rosa, 2021), usar este marco teórico para pensar en las demandas feministas del aborto implica cuestionar la racionalidad del Estado y apelar a puntos de vista alternativos basados en las realidades vividas más allá del discurso y las agendas hegemónicas feministas blancas. 
Por lo tanto, cuerpo-territorio se entiende de manera simultánea como un análisis del 'biopoder' (Foucault, 1978), aquél que se inscribe y ejerce sobre los cuerpos, así como una praxis a través del cuerpo, como los conceptos de soberanía corporal y cuerpo como propiedad y lugar de resistencia (Cabezas González, 2013). Debido a lo anterior, este término se puede usar tanto para conceptualizar la violencia ejercida sobre los cuerpos de las mujeres como acto territorial y colonizador, como para la propia resistencia a la invasión.

De forma simultánea, el cuerpo es un espacio íntimo encarnado y un campo de batalla en donde se juegan intereses políticos encontrados. El concepto cuerpo-territorio evidencia las formas en las que la autonomía se experimenta, espacial y socialmente. Como señala Massey (2005), el cuerpo es el espacio "más cercano" y producto de las interrelaciones sociales que están imbuidas de poder.

Además, el concepto cuerpo-territorio contiene la perspectiva de género, al cuestionar la dicotomía entre espacios públicos (masculinos) y privados (femeninos) (Cruz Hernández, 2016). Así, el espacio corporal está en cambio constante - como sucede con todo espacio- y, en términos de Massey (2005), se encuentra en un estado permanente de negociación y transformación.

A partir de lo anterior, el concepto cuerpo-territorio nos permite pensar en la gobernanza reproductiva como un ejercicio violento y territorial. Hasta ahora, la gobernanza reproductiva se ha conceptualizado como un proyecto biopolítico (Morgan y Roberts, 2012) que pretende la subyugación de los cuerpos y el control de las poblaciones mediante la protección de la vida (Foucault, 1978). Sin embargo, sostengo que en México la gobernanza reproductiva se manifiesta mediante mecanismos necropolíticos que utilizan la violencia para controlar los cuerpos de las mujeres y las niñas.

De acuerdo con Mbembe (2003), quien propone el concepto, la "necropolítica" describe el uso de la violencia y la muerte como mecanismo de gobernanza, en particular en contextos poscoloniales. La biopolítica y la necropolítica existen en una relación simbiótica, en la que el Estado determina quién debe morir para que otros puedan vivir (Agamben, 1998; Mbembe, 2003).

Asimismo, para Mbembe (2003), la raza es el estado de excepción: el factor que separa a cierto sector de la ciudadanía al considerarlo como desechable, despojándolo de los derechos humanos y reduciéndolo a lo que Agamben (1998) denomina nuda vida. Al respecto, autoras feministas lati- 
noamericanas, como Segato (2008) y Valencia (2010), han demostrado que el género, además de la raza, determina cómo se gobiernan los cuerpos.

La filósofa feminista mexicana Sayak Valencia (2010) sostiene que la necropolítica es inherente a lo que llama "capitalismo gore": en lugar de ver la violencia contra el cuerpo de las mujeres como un daño colateral, sostiene que el consumo de cuerpos -a través del comercio sexual, la trata, el trabajo esclavizado, la violencia de género y el feminicidio- es un componente inherente del capitalismo avanzado.

La obra de Valencia hace eco de las aportaciones de la antropóloga feminista Rita Segato (2008), quien se enfoca en cómo se dispone de los cuerpos de las mujeres en una lucha de poder en lo que ella refiere como un Estado paralelo o secundario. Además, afirma que los delitos violentos contra las mujeres son actos performativos que los hombres llevan a cabo para demostrar su poder frente a otros hombres, competidores, actores estatales y la propia sociedad mexicana (Segato, 2008). De forma paradójica, el poder soberano del Estado se ha fortalecido en este proceso por la impunidad y la corrupción (Segato, 2008).

En el contexto del aborto, la filósofa feminista australiana Penelope Deutscher (2008) utiliza la teoría de la gobernanza a través de la muerte para mostrar las formas en las que la legislación punitiva sobre el aborto reduce a las mujeres a su función biológica, su nuda vida.

Retomando el trabajo de estas feministas, utilizo el concepto cuerpo-territorio para pensar en la gobernanza reproductiva como la ocupación violenta de territorios corporales y en el acompañamiento como la defensa de los mismos.

En este sentido, describo las formas en las que el Estado utiliza mecanismos necropolíticos - como la muerte, la violación y la deshumanización- para el control de los cuerpos reproductivos de las mujeres. Por lo tanto, sostengo que el marco de cuerpo-territorio proporciona un terreno teórico pertinente para explorar el papel de la violencia en la política reproductiva en México.

\section{La regulación del aborto: territorios \\ de gobernanza reproductiva}

En México, la legislación sobre el aborto divide al país en jurisdicciones, en las cuales los cuerpos de las mujeres se controlan en diferente medida, circunstancias y formas según su ubicación geográfica. Esta práctica 
reproductiva está regulada por medio de códigos penales estatales, en los que las "causales" definen las circunstancias en las que se puede realizar este procedimiento sin que las mujeres se enfrenten a un castigo penal.

En las treinta y dos entidades federativas de México hay ocho causales circunstanciales, aunque no todas se aplican en cada estado. ${ }^{5}$ Incluso en la Ciudad de México, y más recientemente en los estados de Oaxaca, Hidalgo y Veracruz, en donde el aborto está despenalizado por voluntad de la mujer hasta las doce semanas de embarazo, esta práctica reproductiva todavía está regulada por los códigos penales. De acuerdo con Krauss (2016), la despenalización del aborto no implicó una liberalización total de su práctica; sólo creó una excepción nueva y adicional: la temporalidad.

En 2021, la Suprema Corte de Justicia de la Nación (SCJN) emitió dos fallos en los que declaró inconstitucional la penalización del aborto. Sin embargo, hay reformas a los códigos penales estatales que continúan pendientes y, con base en los antecedentes de otras entidades federativas que han liberalizado su legislación, es muy complicado que en un futuro inmediato existan cambios legislativos que permitan el aborto después del primer trimestre, más allá de las situaciones de "excepción".

De este modo, Deutscher (2008) sostiene que el aborto, en sí mismo, es una especie de estado de excepción, ya que es poco frecuente que se elimine por completo de los códigos penales. Por lo tanto, el aborto, como práctica, existe como una excepción a su propia ilegalidad (Deutscher, 2008).

En este sentido, el problema de la excepcionalidad -la provisión del aborto "legal" en determinadas circunstancias, dentro de ciertos límites o en ubicaciones geográficas específicas- es que se genera un efecto paradójico al reforzar el control estatal sobre los cuerpos de las mujeres. Como sostiene Deutscher (2008), las excepciones pueden constituir un medio de reconfirmar, en una versión intensificada, el régimen de regulación.

Por lo tanto, al permitir el aborto en ciertas circunstancias o en determinados contextos, se intensifica la regulación del cuerpo de las mujeres fuera de estas jurisdicciones o situaciones. El ejemplo de este fenómeno

5 Éstas son las causales y el número de entidades federativas donde son legales: violación (32), imprudencial o culposo (30), peligro de muerte (24), alteraciones graves en el producto (16), salud (16), inseminación artificial no consentida (15), causas económicas (2) y voluntad de la mujer hasta las doce semanas (4). 
son los cambios constitucionales para "proteger la vida desde el momento de la concepción", realizados en dieciséis estados mexicanos como respuesta a la despenalización del aborto en la Ciudad de México. Estas reformas fueron una expresión del conservadurismo de los estados y evidencian un acto de resistencia territorial estatal contra el centralismo político de la Ciudad de México.

Si bien estas reformas no tuvieron un efecto jurídico en las leyes que incluyen las causales de aborto - que siguen vigentes en las constituciones estatales, lo cual fue confirmado con los fallos emitidos por la SCJN en 2021- y aun siendo simbólica la protección de la vida fetal, se generan efectos reales en el trato hacia las mujeres que necesitan realizarse un aborto, en lo que profundizaré más adelante.

De esta forma, la legislación sobre el aborto es un mecanismo de colonización de cuerpos-territorios que tiene efectos violentos. Los cuerpos embarazados son territorios potentes para gobernar poblaciones a través de la violencia, por lo que sostengo que el Estado regula los cuerpos de las mujeres para producir y mantener el poder soberano. La legislación sobre el aborto es un ejercicio territorial pues las entidades estatales marcan límites y encierran espacios a través de los cuerpos.

Durante mi trabajo de campo, entrevisté a acompañantes que asistían a mujeres para realizarse abortos dentro del sistema del Estado (bajo la figura legal de causales) y a quienes operaban al margen de la ley. En la búsqueda de garantizar el cumplimiento del derecho de las mujeres al aborto, negociaron mecanismos legales y regímenes de gobernanza reproductiva violenta. A continuación, describiré las experiencias de dos de ellas.

\section{Territorios, cuerpos y el "Estado paralelo"}

Los cuerpos de las mujeres son controlados como extensiones de otros espacios sociopolíticos de poder y a través de la violencia infligida en los reclamos territoriales del Estado y actores estatales paralelos. Esto es evidente en la experiencia de Asunción, acompañante de un colectivo feminista en Michoacán, región de México que ha sufrido violencia generalizada debido a los conflictos relacionados con la reestructuración neoliberal de las zonas rurales hacia la agricultura industrializada y luego al cultivo ilícito de cannabis y adormidera (Fuentes Díaz y Fini, 2021).

Asunción es universitaria; sus estudios se enfocan en los procesos sociales y el trabajo comunitario. Desde hace dos años, comenzó el 
acompañamiento a mujeres que necesitan realizar un aborto con medicamentos, aunque desde tiempo atrás ha colaborado en colectivos feministas. Nos conocimos en febrero de 2019, durante una reunión sobre género y migración, pues, además de su trabajo en torno al aborto, pertenece a un colectivo que apoya a las mujeres y sus familias desplazadas por la narcoviolencia. Un mes después, sostuvimos una entrevista por videollamada.

En la entrevista, Asunción se refirió a su trabajo de acompañamiento autónomo. El colectivo al que pertenece comparte información sobre cómo interrumpir embarazos en casa, de manera segura y utilizando medicamento. El proceso inicia por teléfono, redes sociales o de manera presencial.

Asunción y sus compañeras recomiendan el uso de misoprostol debido a que puede adquirirse en cualquier farmacia y, por lo general, no se requiere receta médica para su compra. Ellas comparten información sobre la dosis, las contraindicaciones y las señales de alerta. Además, se mantienen en contacto con la mujer mientras realiza la toma del medicamento, con el fin de aclarar sus dudas y mantenerse atentas ante cualquier complicación. La mayoría de las mujeres a las que apoyan son originarias o residentes de Morelia, aunque también han asistido a mujeres de comunidades purépechas y nahuas.

Al exponer su motivación para realizar el acompañamiento, Asunción se refirió al marco de cuerpo-territorio, pues comentó que escuchó este concepto por primera vez cuando trabajaba en una campaña medioambiental para detener la contaminación de un manantial en su localidad. Por lo tanto, estaba acostumbrada a hablar de su activismo ambiental en términos de defensa: la protección de un recurso importante. Un día comprendió que la misma idea se aplicaba a su trabajo sobre el aborto:

Nuestros cuerpos, nuestras cuerpas, son el primer territorio y es amplio esto, la defensa debe ser amplia, incluso para mí estar ahí [en la defensa del manantial] tenía que ver con reconocer el cuerpo, pero luego fue reconocer los cuerpos de las mujeres y las violencias, así más como inmediatas, en las que se puede hacer algo y es seguro que se tiene incidencia. Por ejemplo, eso, que una mujer decida no ser madre y que eso sí se puede resolver. (Entrevista, marzo de 2020) ${ }^{6}$

6 Los corchetes son míos.

Belfrage, M. (2022). Cuerpo-territorio y aborto: propuesta teórica para cuestionar la gobernanza reproductiva violenta. Iberoforum, Revista de Ciencias Sociales, Nueva Epoca, 2(1), 1-19, Artículos, e000194. https://doi.org/10.48102/if.2022.v2.n1.194 Licencia Pública Internacional - CC BY-NC-ND 4.0 
Asunción reconoció en el acompañamiento una forma de tener impacto inmediato en lo que sintió como una lucha interconectada y mucho más amplia contra la violencia hacia los cuerpos de las mujeres. Así, continuó explicando cómo el contexto de su estado influyó en sus ideas sobre el cuerpo-territorio, en particular ante la violencia provocada por las disputas entre los cárteles, el Estado y las autodefensas, ${ }^{7}$ que tuvo impacto directo en los cuerpos de las mujeres.

Como lo describió Asunción, estos actores estaban usando la violación como un método de guerra y vigilaban a las mujeres para que no pudieran interrumpir los embarazos que eran resultado y consecuencia de ataques hacia ellas. Así narró uno de esos casos:

Hemos tenido acompañamientos, concretamente hay uno en el que nosotras tampoco estábamos preparadas con protocolos de seguridad para llevarlo de la manera más cuidadosa, y fue el caso de una chica que había tenido una violación de parte de una persona involucrada en el narco y entonces decide abortar. Era un aborto en tercer trimestre, ella salió del municipio en donde estaba, se trasladó a Morelia. Bueno, pero fue como muy caótico porque estaba huyendo, hay varias cosas donde no tenemos información suficiente y que también para nosotras por cuestiones de seguridad no queremos saber, pero que sigue ahí nebuloso, ¿no? Sin embargo, por supuesto que el asunto del sujeto, el elemento narco, hace distinto todo. (Entrevista, marzo de 2020)

La experiencia de Asunción demuestra que los cuerpos de las mujeres se controlan mediante la violación y la coerción reproductiva, como "necro-herramienta" (Valencia, 2010) en la lucha por el control de territorios políticos, económicos y geográficos más amplios. En este sentido, Segato (2008) sostiene que la violación es un acto alegórico de soberanía; es decir, el perpetrador invade el territorio íntimo de otra persona, desconociendo el estado de derecho, y ocupa el cuerpo como anexo de otros territorios. En otras palabras, los hombres ocupan los cuerpos de las mujeres como producción y extensión del poder sobre el espacio público.

7 Las autodefensas son grupos comunitarios que se arman en defensa contra los cárteles y el ejército. 
Según la experiencia de Asunción, la violencia contra el cuerpo de las mujeres es mucho más que un daño colateral: es una forma específica e intencionada para que grupos rivales de hombres violentos se mantengan en el poder. En este sentido, Valencia (2010) denomina a estas violaciones prácticas como gore y argumenta que son producto de la intersección entre la responsabilización neoliberal de los sujetos y la normatividad racializada y de género de los "machos sostén de la familia" masculinos, lo que da como resultado el "necro-empoderamiento".

Estos actores, abandonados por el Estado y excluidos de la acumulación capitalista "legítima", retoman el uso depredador del cuerpo femenino a través de la violencia y la muerte como parte de la acumulación de riqueza. Por lo tanto, sostengo que participan en una especie de gobernanza reproductiva mórbida y complican la comprensión del control del aborto como mandato exclusivo del Estado, como comentó Asunción:

Que ya no sólo es el Estado, que ya no sólo es la Iglesia, que ya no es el papá o solamente el papá, que ya no solamente es el marido, sino que son estos sujetos, el narco y las autodefensas. (Entrevista, marzo de 2020)

Los cuerpos de las mujeres se vuelven nuda vida en la guerra territorial de estos actores y son gobernados como espacios colonizados en una lógica de acumulación. Como señala Asunción, las acompañantes realizan su trabajo en este contexto caótico en respuesta a los efectos desesperados de la violencia generalizada.

Las mujeres que son víctimas de violencia por parte de integrantes de redes del crimen organizado buscan ayuda del Estado en pocas ocasiones porque saben que es riesgoso. Las autoridades no pueden brindar la protección adecuada a las mujeres que denuncian la violencia y ellas corren el riesgo de ser identificadas si asisten a clínicas públicas; además, cuando lo hacen, a menudo se les niega la atención.

En el caso narrado, el embarazo transitaba el tercer trimestre, más allá del límite "legal" para un aborto en caso de violación sexual. Por su parte, Asunción y sus compañeras del colectivo le brindaron información y apoyo para llevar a cabo el aborto. Éste es un ejemplo de cómo las acompañantes asumen riesgos en contextos en los que el Estado no puede garantizar la seguridad ni los derechos de las mujeres. 
En un entorno donde los cuerpos de las mujeres son un campo de batalla y su vida está en riesgo, el trabajo de las acompañantes para garantizar la autonomía corporal se puede entender como una defensa contra invasiones violentas.

En este sentido, la experiencia de una acompañante del Estado de México evidencia cómo el Estado niega sus responsabilidades y ejerce violencia directa sobre los cuerpos de las mujeres a través del maltrato y de una legislación punitiva.

\section{Violencia estatal territorial y gobernanza reproductiva}

El Estado de México es la franja periurbana que encierra a la Ciudad de México; las áreas poblacionales densas impiden la distinción entre las dos entidades: en una sola calle, en un lado de la acera es la Ciudad de México y en el otro es el Estado de México. Cuando conocí sobre las leyes de aborto por causales, me sorprendió lo absurdo de una situación en la que había partes del área metropolitana en donde esta práctica de salud reproductiva se consideraba legal o ilegal según el lado de la calle en el que se estuviera.

Esperanza es acompañante, estudiante de derecho y madre; desde hace diez años pertenece a una red nacional y gran parte de su trabajo se centra en garantizar que las mujeres puedan abortar en hospitales públicos bajo una de las cuatro causales legales que se contemplan en el estado. ${ }^{8}$ En 2017, cuando comenzó su labor de acompañamiento, asistía aproximadamente a quince mujeres por mes, cantidad que se incrementó conforme se agravaba y profundizaba la violencia de género en el Estado de México y se difundía la información sobre el apoyo que proporciona.

En abril de 2020 entrevisté a Esperanza, quien en esa época apoyaba en promedio a treinta mujeres por mes y en ese momento estaba acompañando tres procesos de aborto. La situación era complicada pues el médico que por lo general la apoyaba había sido llamado para trabajar en un hospital que atendía casos de COVID-19. Ante ese contexto, las mujeres estaban angustiadas y se preguntaban si ella sólo les ofrecía falsas promesas.

Al preguntarle sobre los casos que solía asistir, señaló que la mayoría de los embarazos eran resultado de violación y muchos eran de niñas y

8 Las causales que aplican en el Estado de México son: violación, imprudencial o culposo, peligro de muerte y alteraciones graves en el producto. 
adolescentes de entre diez y dieciséis años. Además, indicó que en el caso de las niñas los perpetradores solían ser familiares o conocidos y que el abuso ocurría en el hogar. ${ }^{9}$ Mientras que las mujeres mayores de dieciocho años solían ser agredidas por desconocidos en lugares públicos: en el transporte, en la calle, al salir del trabajo o en fiestas. ${ }^{10}$

Lo señalado por Esperanza me recordó las afirmaciones de Segato (2008) sobre los feminicidios: los hombres que violan a mujeres y niñas en casa lo hacen porque creen que son su propiedad y aquéllos que lo hacen en el espacio público lo consideran como conquista para marcar el territorio.

En la entrevista, Esperanza comentó que existen dos hospitales a los que enviaba a las mujeres. En uno de ellos, el director se quejó con ella porque les estaba dando "demasiado trabajo" pues en los medios de comunicación Esperanza había nombrado el hospital y estaban sobrepasados de mujeres que buscaban abortos.

En el otro hospital, sólo un médico, quien es contacto personal de la acompañante, realizaba los abortos sin el conocimiento de sus supervisores. En la mayoría de los casos, las mujeres fueron atendidas rápidamente y sin complicaciones. Sin embargo, Esperanza narró casos que no fueron sencillos y que ocurrieron en hospitales en donde no tuvo contacto directo con el personal médico.

Así, describió un caso que sucedió tres años atrás, cuando una enfermera la llamó para decirle que una mujer de diecisiete años había ingresado con fiebre y hemorragia interna después de un legrado hecho en condiciones no esterilizadas. La adolescente había sido violada por su novio y sus amigos en la fábrica en la que trabajaban. Aunque hoy en día la Norma

9 El Estado de México ocupa el primer lugar de nacimientos en niñas y adolescentes de entre diez y catorce años (1137 casos). A nivel nacional, el $46 \%$ de las niñas de diez a catorce años que tuvieron un hijo reportaron que el hombre con el que habían sostenido relaciones sexuales contaba con una edad de entre dieciocho y sesenta y ocho años, lo que evidencia la ocurrencia generalizada de la violencia sexual hacia las niñas, incluidas la coerción y la manipulación (Ipas, 2018).

10 De acuerdo con un análisis de los datos de la Encuesta Nacional sobre la Dinámica de la Relación en los Hogares (ENDIREH) de 2016, Frías (2018) calcula que el 7.8 \% de las mujeres mexicanas ha vivido una violación o intento de violación a lo largo de su vida. En 2020, la Fiscalía General de Justicia del Estado de México registró cinco casos de violación al día, siendo la entidad con mayor número de este tipo de delitos en el país (Secretariado Ejecutivo del Sistema Nacional de Seguridad Pública [SESNSP], 2020). 
Oficial Mexicana 046 señala con claridad que no es requisito que la mujer denuncie para recibir atención médica, en ese entonces era necesaria la aprobación del Ministerio Público. La mujer no había denunciado por temor a represalias y por ello había buscado un aborto con un proveedor clandestino.

La enfermera que llamó a Esperanza estaba preocupada porque el médico se dio cuenta de que la mujer había intentado un aborto "ilegal" y llamó a la policía. Cuando la acompañante llegó al hospital, la mujer sólo había recibido antibióticos, pero no habían hecho nada para detener la hemorragia. Su madre estaba angustiada, pero lo único que el médico le dijo fue: "si su hija logra sobrevivir a esto, irá directamente a la cárcel".

Esperanza habló con el director del hospital y amenazó con llamar a los medios de comunicación si no hacían todo lo posible para salvar a la mujer. Días después, cuando el personal médico aún no había logrado controlar la hemorragia, la acompañante llamó a su contacto personal de otro hospital, quien acudió y exigió su traslado.

La vida de la mujer se salvó, pero requirió una cirugía para extirparle el útero, lo que fue muy difícil de aceptar para su madre, quien señaló que quitarle la capacidad de tener hijos era "mutilarla de por vida".

La experiencia de Esperanza en hospitales públicos demuestra que, aun cuando algunos abortos se consideren legales según las condiciones suscritas por la ley, existen obstáculos importantes para que se otorgue la atención adecuada. Como acompañante, Esperanza es un elemento clave para que el Estado cumpla con sus obligaciones, incluso en un contexto en el que un imperativo ético y un compromiso profesional deberían obligar al personal médico a salvar la vida de una mujer. Esto ocurrió en el caso narrado, en el que la intervención de la acompañante fue fundamental para la sobrevivencia de la mujer.

La experiencia de Esperanza demuestra lo que las activistas feministas denuncian a través de sus gritos sobre el Estado-violador: muchas veces es el propio Estado el que ejerce violencia en los cuerpos de las mujeres. Si bien la función biopolítica de las leyes punitivas sobre el aborto -es decir, "proteger la vida" intrauterina- es evidente por sí misma, el Estado también promulga una especie de necropolítica, ya que reduce a las mujeres a la nuda vida, sin derechos y sin humanidad.

De esta forma, el control reproductivo por parte del Estado se justifica transformando los cuerpos de las mujeres, de manera simultánea, en ob- 
jetos con capacidad reproductiva y una amenaza para la "(pre)vida" dentro del útero (Deutscher, 2008). Es decir, se regulan los cuerpos de las mujeres como territorios que dan vida y que la ponen en peligro.

Al criminalizar el aborto, la mujer muere como sujeto despojado de derechos para que se puedan instigar los derechos del feto (Deutscher, 2008). Si bien para Deutscher (2008) esta muerte es quizás ontológica, en la historia de Esperanza el riesgo de muerte física fue real, pues la mujer que quedó sangrando en la cama de un hospital público fue despojada de cualquier subjetividad que no fuera la de una criminal o "soberano siniestro".

En este sentido, la violación significó que el aborto de la mujer era una excepción y legalmente tenía derecho a interrumpir el embarazo. Sin embargo, para el personal médico fue más importante reestablecer la soberanía estatal mediante acciones punitivas que salvar la vida de la mujer.

Al hacerlo, la humanidad de la mujer se borró, además de todo el contexto en el que ocurrió el embarazo, incluida la violación y la invasión violenta de su cuerpo. Por lo tanto, el Estado fue cómplice de la violación y la mutilación.

\section{Conclusiones}

Las experiencias de Asunción y Esperanza narran la lucha por la autonomía corporal de las mujeres bajo condiciones complejas de violencia. La ley, aún con restricciones, debe proteger el derecho de las mujeres a interrumpir los embarazos resultados de violencia. Sin embargo, el hecho de que la ley sólo permite este procedimiento bajo ciertas excepciones intensifica la gobernanza reproductiva de los cuerpos de las mujeres.

En este ensayo, he argumentado que el concepto cuerpo-territorio ofrece un marco para evidenciar y analizar las luchas por el poder que se desarrollan a través de los cuerpos de las mujeres en México.

Los cuerpos de las mujeres se gobiernan como territorios productores de vida para ser conquistados en proyectos soberanos de poder sobre espacios políticos más amplios. La violencia se usa como método de invasión y control de los cuerpos-territorios, tanto por el Estado como por los actores estatales paralelos (Segato, 2008). Por medio de la violencia, legislativa y física, las mujeres son despojadas de cualquier derecho o subjetividad más allá de su función reproductiva.

En este contexto, el acompañamiento se constituye como la defensa de los cuerpos-territorios de las mujeres. Las acompañantes asumen la 
tarea de garantizar el acceso al aborto cuando el Estado es incapaz de hacerlo e incluso cuando es perpetrador de la violencia. Por lo que sostengo que el acompañamiento del aborto restaura la subjetividad humana de las mujeres, que implica mucho más que garantizar su "derecho a decidir".

\section{Referencias bibliográficas}

Agamben, G. (1998). Homo sacer: Sovereign power and bare life. Stanford University Press.

Cabezas González, A. (2013). Cuerpos que importan en las geometrías del poder. XV Encuentro de Latinoamericanistas Españoles, Madrid, España. https://halshs.archives-ouvertes.fr/halshs-00875571/document

Cabnal, L. (2010). Acercamiento a la construcción de la propuesta de pensamiento epistémico de las mujeres indígenas feministas comunitarias de Abya Yala. En Asociación para la Cooperación con el Sur, Feminismos diversos: El feminismo comunitario (pp. 11-25). ACSUR-Las Segovias.

Craven, C. y Davis, D. A. (2013). Feminist activist ethnography counterpoints to neoliberalism in North America. Lexington Books.

Cruz Hernández, D. T. (2016). Una mirada muy otra a los territorios-cuerpos femeninos. Solar: Revista de Filosofía Iberoamericana, 12(1), 35-46.

Curiel, O. (2015). Construyendo metodologías feministas desde el feminismo decolonial. En I. Mendia Azkue, M. Luxán, M. Legarreta, G. Guzmán, I. Zirion y J. Azpiazu Carballo (eds.), Otras formas de (re) conocer: Reflexiones, herramientas y aplicaciones desde la investigación feminista (pp. 45-60). Hegoa.

Deutscher, P. (2008). The inversion of exceptionality: Foucault, Agamben, and "reproductive rights". South Atlantic Quarterly, 107(1), 55-70.

Foucault, M. (1978). The history of sexuality. Vol. 1: An Introduction. Pantheon Books.

Frías, S. (2018). Violación e intento de violación de mujeres, patrones de búsqueda de ayuda y denuncia. Un análisis a partir de la ENDIREH 2016. Papeles de Población, 24(95), 237-272.

Fuentes Díaz, A. y Fini, D. (2021). Neoliberalism in the grey area: Community defense, the State, and organized crime in Guerrero and Michoacán. Latin American Perspectives, 48(1), 84-102.

Ipas México (2018). Violencia sexual y embarazo infantil en México: Un problema de salud pública y derechos humanos. 
Krauss, A. (2016). Temporalities of exception: Abortion law, feminist advocacy, and clinical intervention in Mexico City [tesis doctoral, Johns Hopkins University]. https://jscholarship.library.jhu.edu/ handle/1774.2/60721

Lamas, M. (2015). El largo camino hacia la ILE: Mi versión de los hechos. Universidad Nacional Autónoma de México, Programa Universitario de Estudios de Género.

Massey, D. (2005). For space. Sage.

Mbembe, A. (2003). Necropolitics (L. Meintjes, trad.). Public Culture, 15(1), 11-40.

Mohanty, C. T. (2003). "Under Western Eyes" revisited: Feminist solidarity through anticapitalist struggles. Signs, 28(2), 499-535.

Morgan, L. M. y Roberts, E. F. S. (2012). Reproductive governance in Latin America. Anthropology \& Medicine, 19(2), 241-254.

Paredes, J. (2008). Hilando fino desde el feminismo comunitario. Comunidad Mujeres Creando Comunidad.

Rosa, C. (2021, 17 de mayo). Yuderkys Espinosa: "El feminismo (blanco) es un programa totalmente moderno y si es moderno es racista". Revista La Brújula. Periodismo feminista. https://revistalabrujula.com/2021/05/17/yuderkys-espinosa-el-feminismo-blanco-es-un-programa-totalmente-moderno-y-si-es-moderno-es-racista/

Secretariado Ejecutivo del Sistema Nacional de Seguridad Pública (2020). Información sobre la violencia contra las mujeres (Incidencia delictiva y llamadas de emergencia 911). https://www.gob.mx/sesnsp/es/

Segato, R. L. (2008). La escritura en el cuerpo de las mujeres asesinadas en Ciudad Juárez: Territorio, soberanía y crímenes de segundo estado. Debate Feminista, 37, 78-102.

Valencia, S. (2010). Capitalismo Gore. Melusina.

Vargas, V. (2017). Some thoughts on new epistemologies in Latin American feminisms. En W. Harcourt (ed.), Bodies in Resistance (pp. 295-309). Springer. 